\title{
CUBIU: FRUTO AMAZÔNICO PREVINE OXIDAÇÃO DE LIPÍDEOS, PROTEGENDO CONTRA A FORMAÇÃO DE ATEROMA
}

Nathália Cardoso de Afonso Bonotto; Laboratório de Biogenômica, Departamento de Morfologia, Universidade Federal de Santa Maria; nathaaliab23@gmail.com

Greice Franciele Montagner; Universidade Regional do Noroeste do Estado do Rio Grande do Sul; greicemontagner@gmail.com

Ivana Beatrice Mânica da Cruz; Programa de Pós-Graduação em Gerontologia, Universidade Federal de Santa Maria; ibmcruz@hotmail.com

Ednea Aguiar Maia-Ribeiro; Fundação Universidade Aberta da Terceira Idade do Amazonas; edneaamribeiro@hotmail.com

Euler Esteves Ribeiro; Universidade Aberta da Terceira Idade/Universidade do Estado do Amazonas; unatieuler@gmail.com

Rômulo Pillon Barcellos; Universidade de Passo Fundo; romulo1604@hotmail.com

Fernanda Barbisan; Programa de Pós-Graduação em Gerontologia, Universidade Federal de Santa Maria; fernandabarbisan@gmail.com

\section{RESUMO}

Introdução: $\mathrm{O}$ envelhecimento é um fator de risco para o desenvolvimento de doenças cardiovasculares, sendo a aterosclerose um exemplo destas patologias, a qual se caracteriza pelo acumulo de gordura, especialmente lipoproteínas de baixa densidade (LDL), nas artérias. Sabendo que o acumulo e a oxidação do LDL por espécies reativas de oxigênio (EROs) fazem parte do mecanismo de iniciação e progressão das placas de ateromas, e que fragmentos da placa podem se desprender, ocluindo as artérias e gerando falta de oxigênio e morte de tecidos, como ocorre no infarto do miocárdio, que é a principal causa de morte no mundo, se torna necessária a busca por alimentos que possam diminuir a oxidação do LDL. O cubiu (Solanum sessiliflorum), fruto amazônico conhecido por seu forte poder antioxidante, poderia ser utilizado no combate às EROs que oxidam o LDL. Objetivo: Investigar o efeito do cubiu sobre a oxidação do colesterol LDL. Métodos: Preparação do extrato hidroalcóolico do cubiu proveniente de Maués- AM. Sangue foi coletado e amostras de plasma contendo LDL foram incubadas com um agente oxidante e com o extrato hidroalcólico nas concentrações de 3 a $300 \mu \mathrm{g} / \mathrm{mL}$. Resultados: Diferentes concentrações de cubiu foram capazes de controlar processos de oxidação de LDL no plasma in vitro. Conclusão: Nossos resultados sugerem que o extrato de cubiu tem potencial ação protetora frente a oxidação do LDL, indicando capacidade de prevenção da formação de ateromas. Mais estudos precisam ser realizados para a confirmação desta hipótese e possível indicação do cubiu como suplemento alimentar.

Palavras-chave: Envelhecimento; Doenças cardiovasculares; Espécies reativas de oxigênio.

Agradecimentos: Capes, CNPq, FAPEAM. 